\title{
New Consistency Model Based on Inertial Operating Speed Profiles for Road Safety Evaluation
}

\author{
David Llopis-Castelló1; Francesco Bella²; Francisco Javier Camacho-Torregrosa ${ }^{3}$; \\ and Alfredo García ${ }^{4}$
}

\begin{abstract}
Road crashes are mainly caused by three concurrent factors: infrastructure, vehicles, and human factors. The interaction between infrastructure and human factors leads to the concept of geometric design consistency, which can be defined as how drivers' expectations and road behavior fit. This paper presents a new global consistency model based on the difference between the inertial operating speed profile ( $\left.V_{i}\right)$ and the operating speed profile $\left(V_{85}\right)$. The first is calculated as the weighted average speed of the previous road section and represents drivers' expectations, whereas the second represents road behavior. A set of 71 homogeneous two-lane rural road segments located in Italy were used in the calibration of the model. As a result, a safety performance function based on this new consistency model was proposed to estimate the number of crashes on an entire road segment. Finally, the new model was compared with previous global consistency models, concluding that the new consistency parameter better explains the phenomenon than the previous ones. Therefore, the new consistency model is a useful tool for engineers that allows estimation of the number of crashes and incorporates road safety into the geometric design of both new two-lane rural roads and improvements to existing highways. DOI: 10.1061/JTEPBS.0000126. (C) 2018 American Society of Civil Engineers.
\end{abstract}

\section{Introduction}

More than 1.2 million people die every year on the world's roads, making road traffic injuries a leading cause of death globally. This costs governments approximately 3\% of their GDPs (WHO 2015). The majority of fatalities occur on rural roads. Specifically, in Italy, $48 \%$ of all road accidents took place on this type of road between 2011 and 2013 (European Transport Safety Council 2015).

Road crashes may be caused by several concurrent factors, with infrastructure, vehicle, and human factors the most important ones. Particularly, the infrastructure factor is behind over $30 \%$ of road crashes (Treat et al. 1979). In fact, accidents tend to concentrate at certain road elements. For this, infrastructure and its interaction with the human factor have been widely studied for geometric design consistency. Road design consistency can be defined as how drivers' expectations and road behavior fit. In this regard, a consistent road means that the road user is not surprised while driving along it. On the contrary, an inconsistent road design produces surprises for drivers, leading to anomalous behavior and increasing the likelihood of crash occurrences.

There are several methods to evaluate geometric design consistency: operating speed, vehicle stability, alignment indices, and

${ }^{1} \mathrm{Ph} . D$. Student, Highway Engineering Research Group, Universitat Politècnica de València, Camino de Vera, s/n., 46022 Valencia, Spain (corresponding author). ORCID: https://orcid.org/0000-0002-9228-5407. E-mail: dallocas@doctor.upv.es

${ }^{2}$ Associate Professor, Dept. of Engineering, Roma Tre Univ., Via Vito Volterra, 62, 00146 Rome, Italy. E-mail: francesco.bella@uniroma3.it

${ }^{3}$ Assistant Professor, Highway Engineering Research Group, Universitat Politècnica de València, Camino de Vera, s/n., 46022 Valencia, Spain. E-mail: fracator@tra.upv.es

${ }^{4}$ Professor, Highway Engineering Research Group, Universitat Politècnica de València, Camino de Vera, s/n., 46022 Valencia, Spain. E-mail: agarciag@tra.upv.es

Note. This manuscript was submitted on May 23, 2017; approved on September 22, 2017; published online on January 18, 2018. Discussion period open until June 18, 2018; separate discussions must be submitted for individual papers. This paper is part of the Journal of Transportation Engineering, Part A: Systems, (C) ASCE, ISSN 2473-2907. driver workload (Gibreel et al. 1999). The use of operating speed to determine consistency is the most commonly used method. Operating speed is defined as the 85 th percentile of the speed distribution for passenger cars under free-flow conditions with no environmental restrictions $\left(V_{85}\right)$. This speed can be estimated by the means of operating speed models.

Operating speed consistency can be analyzed using local and global assessments. Local models focus on localized issues, such as sudden speed reductions or high differences between the design and operating speeds. Those models are ideal to detect where road accidents are more likely to occur. On the other hand, global consistency models examine the overall speed variation throughout an entire road segment. Even though they do not indicate where accidents are prone to take place, they can be introduced into a safety performance function (SPF) to estimate the number of crashes on the entire road segment.

The most widely used local method was developed by Lamm et al. (1999). They presented two design consistency criteria related to operating speed. Criterion I focuses on disparities between operating and design speeds, and Criterion II examines operating speed differences between successive elements. Lamm et al. established thresholds for both criteria, distinguishing between good, fair, and poor consistency based on average accident rates observed at several alignment layouts.

There are some other local consistency criteria, such as Leisch and Leisch (1977), Kanellaidis et al. (1990), and García et al. (2013b). The first one proposed the following three consistency criteria:

- The difference between design speeds of two consecutive road segments should not exceed $10 \mathrm{mi} / \mathrm{h}$;

- The difference between the operating speeds for passenger cars of two consecutive road geometric elements should not exceed $10 \mathrm{mi} / \mathrm{h}$; and

- The difference between operating speeds for passenger vehicles and trucks should not exceed $10 \mathrm{mi} / \mathrm{h}$.

Kanellaidis et al. (1990) suggested that a consistent road design could be achieved if the operating speed difference between two consecutive road geometric elements were lower than $10 \mathrm{~km} / \mathrm{h}$. 
Also, finally, García et al. (2013b) presented a novel approach to calculating drivers' expectations and behavior. For them, drivers' expectations at a certain location can be estimated considering the inertial operating speed $\left(V_{i}\right)$, which is the average operating speed of the previous $1,000-\mathrm{m}$ road segment. On the other hand, road alignment behavior can be estimated with the operating speed $\left(V_{85}\right)$. They defined the inertial consistency index (ICI) as the difference between $V_{i}$ and $V_{85}$. Therefore, the larger this index, the more different drivers' expectations and road behavior are; thus, accidents are more likely to occur.

Related to this, several authors have analyzed the influence of the preceding road section on operating speed of two-lane rural roads considering the geometric characteristics of the preceding element and the environmental or desired speed (Praticò and Giunta 2012). As a conclusion, all studies highlighted the importance of considering the conditions of the preceding alignment to better estimate the operating speed on curves and tangents. Therefore, drivers' behavior at a particular point of the alignment is influenced by the expectations generated from the preceding road section.

Another type of consistency criteria is global consistency models. These kind of models were first introduced by Polus and Mattar-Habib (2004). They used two parameters to evaluate geometric design consistency: relative area $\left(R_{a}\right)$ and operating speed dispersion $(\sigma)$. The first parameter was defined as the area bounded by the operating speed profile and the average operating speed, divided by the length of the road segment. Later, they updated their model by adding speed dispersion induced by heavy vehicles as a surrogate measure to vertical alignment (Mattar-Habib et al. 2008).

Related to this, Garach et al. (2014) developed a new consistency model based on the same parameters for two-lane rural roads in Spain. In addition, they stated that the Polus and Mattar-Habib model was quite conservative because some road sections were classified as poor according to the global model while presenting fair consistency according to $R_{a}$ and $\sigma$.

Camacho-Torregrosa (2015) developed another global consistency model considering two operational parameters: the average operating speed and the average deceleration rate. He examined how the selection of the road segment has an important role in the estimation of road crashes. Several SPFs were developed accordingly.

In this regard, there are several previous studies that have analyzed the relationship between design consistency and road safety. Anderson et al. (1999) analyzed the relationship between traffic volume, curve length, and different consistency parameters such as speed reduction or average radius and crashes using Poisson, negative binomial, and lognormal regression analysis.

$\mathrm{Ng}$ and Sayed (2004) and Awatta et al. (2006) calibrated several models to quantify road safety from the study of the relationships between different individual and combined consistency measures and the actual collision experience using regression analysis.

Cafiso et al. (2010) developed a comprehensive accident model for two-lane rural roads considering the exposure, geometry, consistency (curvature ratio, operating speed dispersion, and number of speed differentials higher than $10 \mathrm{~km} / \mathrm{h}$ ), and context factors (driveway density and roadside hazard rating).

Quddus (2013) concluded that average speeds are not associated with crash rates for unusual road conditions, such as high traffic volume or more than one lane per direction. Nevertheless, speed variations did present a significant influence and a positive trend on crash occurrence. Wu et al. (2013) analyzed the number of crashes by means of the difference between operating speed and inferred design speed. They concluded that there is a statistically significant positive association between consistency and safety.
Finally, Montella et al. (2008) and Montella and Imbriani (2015) highlighted that design consistency measures significantly affected road safety, not only on two-lane rural highways, but also on motorways. In the first study, they developed several crash prediction models for total crashes and severe (fatal plus all injury) crashes on Italian rural motorways incorporating some variables related to consistency, such as operating speed reduction. In the second study, they calibrated different SPFs from consistency variables such as the speed reduction between successive elements and the inertial speed considering the previous $5 \mathrm{~km}$.

However, the selection of the road segment turns out to be crucial to applying global consistency models. Selected road segments must be homogeneous because the results depend on its selection (Cafiso et al. 2010; Camacho-Torregrosa 2015; Resende and Benekohal 1997). There are three different complementary criteria to determine them:

- Examining cross-sectional or important traffic variations;

- German method based on curvature change rate $(C C R)$ variations; and

- Analyzing users' operational behavior by means of the inertial operating speed (Garcia et al. 2013a).

The way the inertial operating speed is calculated was revealed as a crucial factor. This factor aims to represent drivers' expectations; therefore, it should correctly reflect their previous behavior. Traditionally, this speed has been calculated based on distance, considering different lengths and extracting a simple average. However, this does not match the drivers' expectation acquirement process, which is related to short-term memory (STM).

STM is the memory system that contains moment-to-moment conscious thoughts and perceptions. According to Revlin (2012), the capacity of STM increases with a person's age until it reaches a maximum in young adulthood. As long as a person able to rehearse, or pay attention to the information in STM, it can reside there indefinitely. However, without rehearsal, STM gradually declines and the information is lost in approximately 18 s (Revlin 2012). As a result, drivers do not recall all locations of the previous road section with the same intensity, so speed-based expectancies of drivers should be more based on the final part of the driven section rather than on one from the beginning. Therefore, estimating $V_{i}$ as a weighted average operating speed would be more suitable to represent drivers' expectations.

This paper presents a new global consistency model based on the geometric design consistency concept for Italian two-lane rural roads. In a previous study, a new local consistency index was developed based on the difference between inertial operating speed and operating speed (García et al. 2013b). Because of the good behavior of this index, different consistency parameters have been defined from the difference between the inertial operating speed profile and operating speed profile to develop a new global consistency model. These parameters will be assessed through SPFs to identify which parameter better estimates the number of crashes.

\section{Objectives and Hypotheses}

The main objective of the study is to develop a global consistency model based on how driver's expectancies and road behavior differ. This difference will be studied considering the inertial operating speed profile $\left(V_{i}\right)$ and the operating speed profile $\left(V_{85}\right)$. The difference between $V_{i}$ and $V_{85}$ will be considered a surrogate measure to geometric design consistency. In this regard, this research aims to determine how $V_{i}$ should be calculated comparing its difference with $V_{85}$ toward the number of crashes. As a result, a safety performance function based on the new consistency model will be 
obtained to estimate the number of crashes on an entire road segment.

The underlying hypothesis is that an inertial operating speed profile defined as the weighted average operating speed of the preceding road section is a surrogate measure to the actual behavior of the driver, who better recalls the last meters driven. In addition, the greater the difference between $V_{i}$ and $V_{85}$, the lower the consistency and higher the likelihood of crash occurrence.

\section{Methodology and Data Description}

\section{Methodology}

This research develops a new global geometric design consistency model based on the relationship between the operating speed behavior and road crashes. Continuous operating speed profiles were used in the analysis.

A large set of two-lane rural road sections was selected in Italy. The geometry of each road segment was recreated to calculate the operating speed profile. In this regard, the operating speed model developed by Marchionna and Perco (2008) was used, which was calibrated from Italian two-lane rural roads. In addition, traffic and crash data were obtained to identify the traffic volume and the number of crashes for each homogeneous road segment.

Finally, different safety performance functions were calibrated to identify the best consistency parameter and how inertial operating speed should be calculated.

\section{Road Segments}

A total of 48 road sections located in Italy were selected for the study. These produced 71 homogeneous road segments according to traffic volume, cross-section variations, major intersections, and curvature change rate $(C C R)$.

First, road segments were divided into sections with similar traffic volume and cross-sections. Major intersections also have an effect on drivers' expectancies, so they were also considered for division. Finally, each road section was divided according its geometric behavior using the curvature change rate. Fig. 1 shows how this last step was carried out: a profile of the cumulative absolute deflection angle versus the road station must be plotted. Hence, homogeneous road segments can be distinguished according to

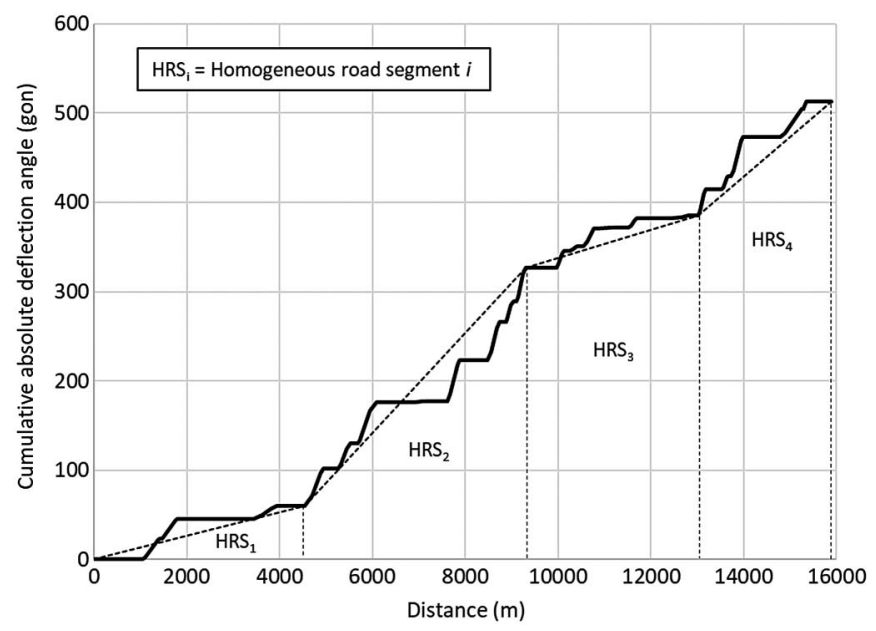

Fig. 1. Determination of homogeneous road segments similar $C C R$ behavior. $C C R$ is defined as the rate between the sum of the absolute deflection angles per length unit $(\mathrm{km})$.

They were two-lane rural roads with annual average daily traffic $(A A D T)$ volume ranging from 1,319 to $19,577 \mathrm{vpd}$. Their length varied from 1,915 to $19,325 \mathrm{~m}$, and their longitudinal grade did not exceed $5 \%$. Regarding their cross-sections, the lane width ranged from 3.00 to $3.50 \mathrm{~m}$, and the shoulder width varied from 0.50 to $1.50 \mathrm{~m}$.

\section{Traffic and Crash Data}

Traffic volume and crash data were provided by the Azienda Nazionale Autonoma delle Strade (ANAS) and the Automobile Club Italia (ACI), respectively. Thus, the $A A D T$ and the number of crashes with victims were identified for each homogeneous road segment. Regarding this, accidents with victims refers to injury and fatal crashes, with the exclusion of property damage only (PDO) accidents.

$A A D T$ was defined as the average traffic volume from 2012 to 2015. Only crashes with victims were considered between 2005 and 2014. Only crashes because of road alignment were considered, so all crashes caused by vehicles entering the road at an intersection were removed from the analysis. As a result, a total of 2,080 accidents were reported, which involved 202 fatalities and 3,701 injured.

Table 1 shows a statistical summary of the main characteristics of the homogeneous road segments used in this study.

\section{Horizontal Alignment and Speed Profiles}

Operating speed profiles for all road segments were obtained by means of the horizontal geometry, which was recreated by means of the methodology proposed by Camacho-Torregrosa et al. (2015).

Operating speed profiles were obtained for each road segment using the operating speed profile model calibrated by Marchionna and Perco (2008) for Italian two-lane rural roads. The model takes into consideration the general character of the horizontal alignment by estimating the desired speed using the curvature change rate (Table 2).

\section{Inertial Operating Speed}

Finally, inertial operating speed profiles were calculated from the operating speed profile of each road segment. This speed aims to represent the driver's expectations, which depends on the shortterm memory that is gradually in decline (Revlin 2012).

Therefore, the inertial operating speed at each point of the alignment was defined as the weighted average operating speed of the preceding road section.

Different distances and weighting distributions were considered to estimate the inertial operating speed. Distances $(L)$ were considered between 100 and $1,500 \mathrm{~m}$ with a step of $100 \mathrm{~m}$, whereas four weighting distributions were proposed: constant, linear, convex parabolic, and concave parabolic (Fig. 2).

The constant distribution provided the average speed. The weighting factor for the other distributions ranged from 0 to 1 , increasing as the station got closer to the critical section. Particularly,

Table 1. Statistical Summary of the Homogeneous Road Segments

\begin{tabular}{lccrr}
\hline Variable & Minimum & Maximum & Average & Deviation \\
\hline Length $(\mathrm{m})$ & 1,915 & 19,325 & $7,839.54$ & $4,130.32$ \\
$C C R(\mathrm{gon} / \mathrm{km})$ & 1.36 & 443.47 & 91.46 & 101.91 \\
AADT $(\mathrm{vpd})$ & 1,319 & 19,577 & $6,874.31$ & $4,461.53$ \\
Accidents & 2 & 184 & 29.30 & 35.06 \\
\hline
\end{tabular}


Table 2. Operating Speed Profile of Marchionna and Perco

\begin{tabular}{lc}
\hline Type of road element & \multicolumn{1}{c}{ Equation } \\
\hline Tangent & $V_{\text {des }}=123.54-2.79 \cdot C C R^{0.47}(\mathrm{~km} / \mathrm{h})$ \\
Curve $(C C R<30$ gon $/ \mathrm{km})$ & $V_{85}=124.08-\frac{563.78}{\sqrt{R}}(\mathrm{~km} / \mathrm{h})$ \\
$\begin{array}{l}\text { Curve }(30 \text { gon } / \mathrm{km} \\
<C C R<80 \text { gon } / \mathrm{km})\end{array}$ & $V_{85}=118.11-\frac{510.56}{\sqrt{R}}(\mathrm{~km} / \mathrm{h})$ \\
Curve $(80$ gon $/ \mathrm{km}$ & $V_{85}=111.65-\frac{437.44}{\sqrt{R}}(\mathrm{~km} / \mathrm{h})$ \\
$<C C R<160$ gon $/ \mathrm{km})$ & \\
$\begin{array}{l}\text { Curve } \\
(C C R>160 \text { gon } / \mathrm{km})\end{array}$ & $V_{85}=100.85-\frac{346.62}{\sqrt{R}}(\mathrm{~km} / \mathrm{h})$ \\
Acceleration rate & $a=1.328-0.159 \cdot \ln (R)\left(\mathrm{m} / \mathrm{s}^{2}\right)$ \\
Deceleration rate & $d=1.757-0.222 \cdot \ln (R)\left(\mathrm{m} / \mathrm{s}^{2}\right)$ \\
\hline
\end{tabular}

Note: $C C R=$ curvature change rate of the road segment $(\mathrm{gon} / \mathrm{km})$; $R=$ radius of the curve; $V_{\text {des }}=$ desired speed on tangents $(\mathrm{km} / \mathrm{h}) ; V_{85}=$ operating speed on curve $(\mathrm{km} / \mathrm{h})$.

the vertices of the convex and concave parabolic distributions were in 1 and 0 , respectively. The equations of these distributions are shown in Fig. 2, where $s_{j}$ is the actual station in meters, $w_{j}$ is the weighting factor for every station in this station, and $s_{o}$ and $s_{f}$ are the initial and final station in meters.

Then, the inertial operating speed [Eq. (1)] is the weighted average speed of the last $L$ meters according to a particular weighting distribution

$$
V_{i, k}=\frac{\sum w_{j} \cdot V_{85, j}}{\sum w_{j}}
$$

where $V_{i, k}=$ inertial operating speed $(\mathrm{km} / \mathrm{h})$ at the point $k ; V_{85, j}=$ operating speed at the point $j$; and $w_{j}=$ weighting factor at the point $j$.

As a result, 60 (15 values of length $\times 4$ weighting distributions) inertial operating speed profiles were developed for each road segment. The main objective was to identify which length and weighting distribution better represents drivers' expectations. Fig. 3 shows the inertial operating speed profile considering a length of $1,000 \mathrm{~m}$ and a linear distribution. As an example, the inertial operating speed calculation is shown for the station $2,125 \mathrm{~m}$. The horizontal side of the orthogonal triangle represents the station difference $(1,000 \mathrm{~m})$, whereas the vertical one is related to the weighting distribution, which linearly ranged from 0 to 1 .

\section{Consistency Parameters}

Different consistency parameters were defined, considering several variables derived from the difference between the inertial operating speed profile and operating speed profile (Fig. 4). According to this definition, a positive value of this difference means drivers' expectations are violated because drivers' speeds are lower than the expected speed. This situation leads to a higher likelihood of crash occurrence.

For a particular road segment, the following variables are calculated (Fig. 5):

- $A(\mathrm{~m} \cdot \mathrm{km} / \mathrm{h})$ : area bounded by the difference between $V_{i}$ and $V_{85}$, and the $x$ axis;

- $L(\mathrm{~m})$ : length of the road segment;

- $\sigma(\mathrm{km} / \mathrm{h})$ : standard deviation of the difference between $V_{i}$ and $V_{85}$;

- $A(+)(\mathrm{m} \cdot \mathrm{km} / \mathrm{h}):$ area bounded by the difference between $V_{i}$ and $V_{85}$ considering only the positive differences;
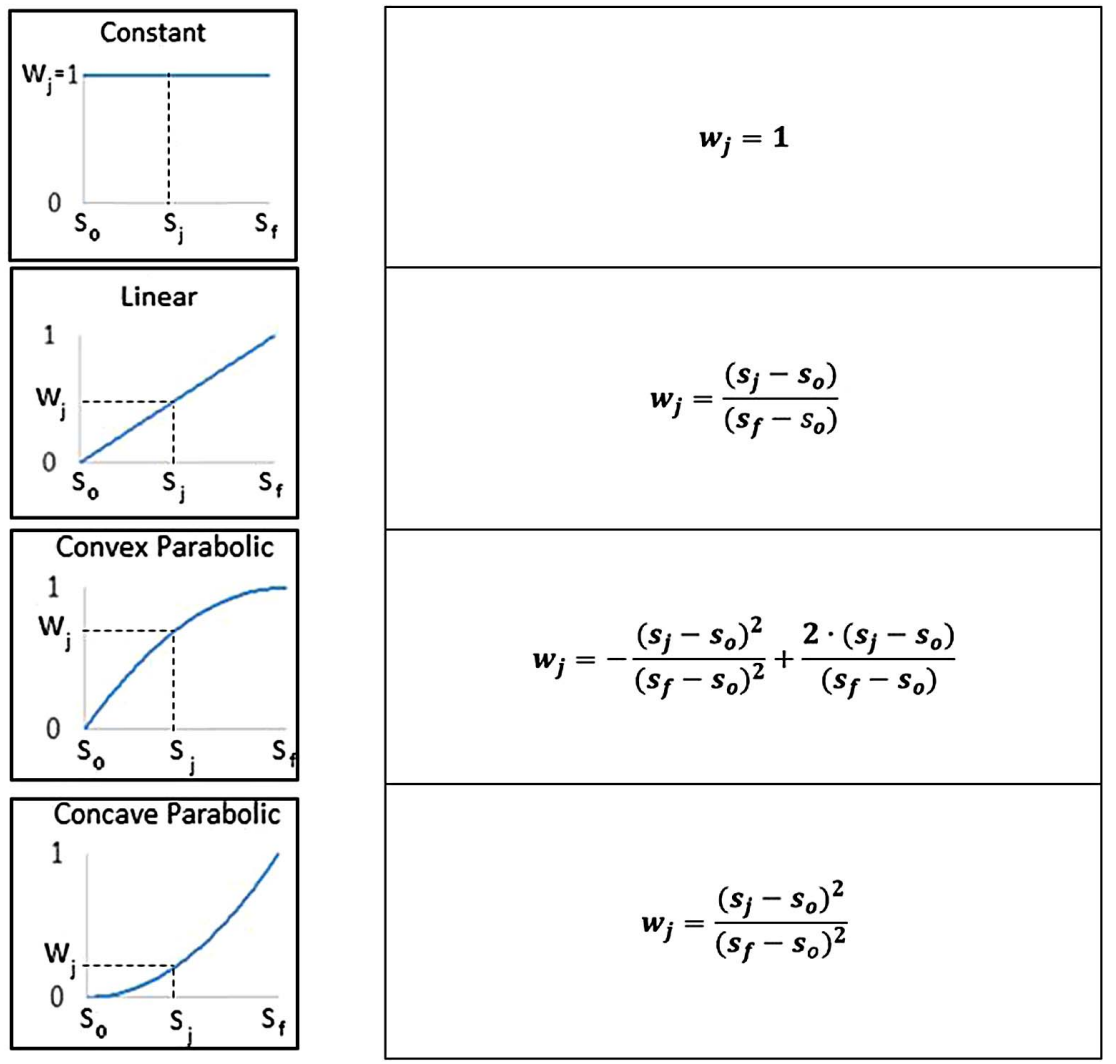

Fig. 2. Weighting distributions 


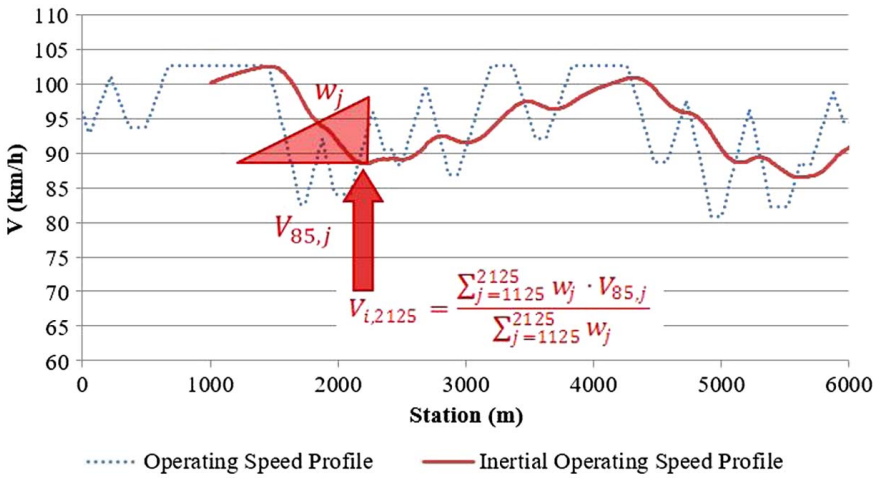

Fig. 3. Speed profiles

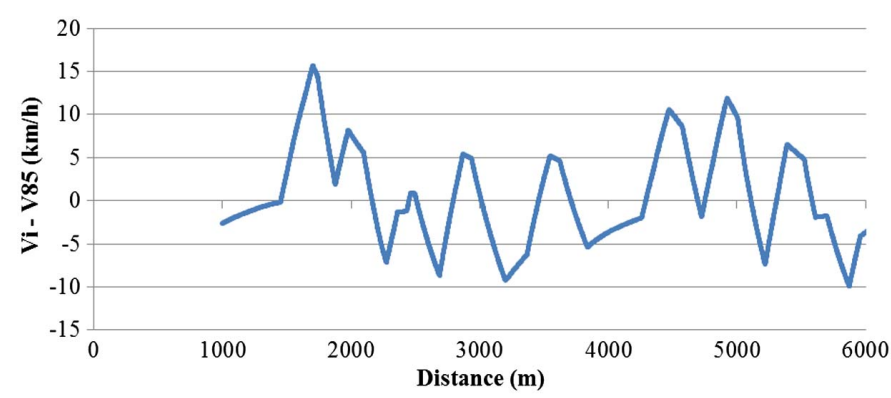

Fig. 4. Difference between $V_{i}$ and $V_{85}$

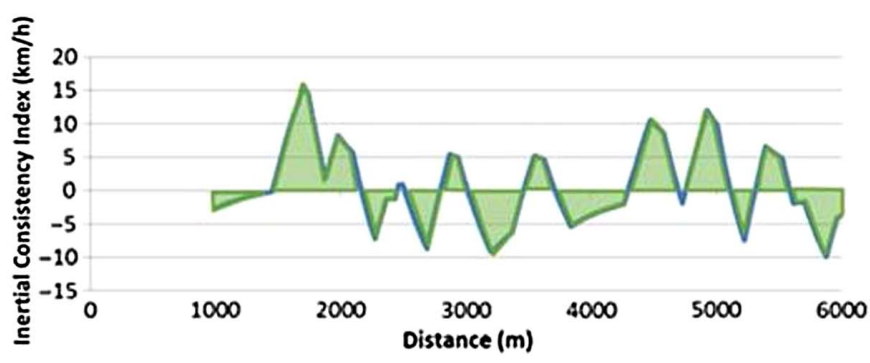

(a) $\quad-1000 \mathrm{~m} /$ Weighted Average Speed - Linear

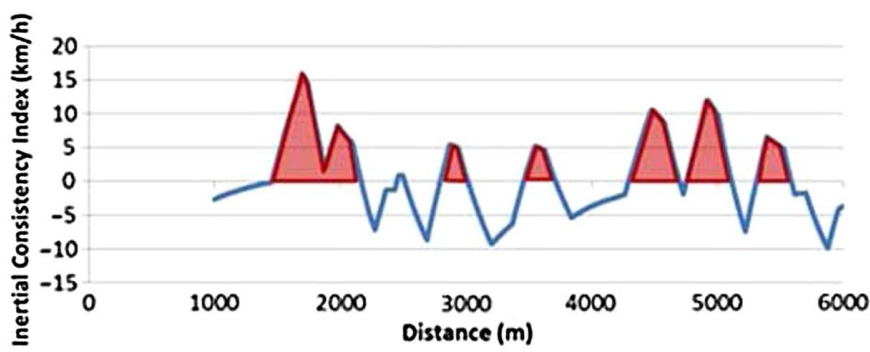

(b) $\quad-1000 \mathrm{~m} /$ Weighted Average Speed - Linear

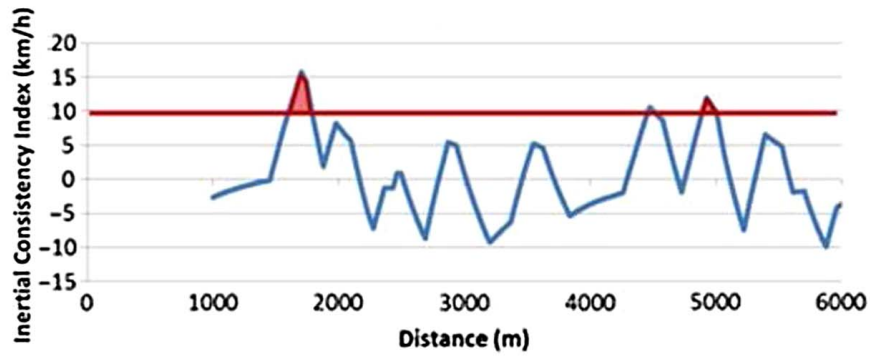

(c) $\quad-1000 \mathrm{~m} /$ Weighted Average Speed - Linear

Fig. 5. Consistency variables: (a) $A, L$, and $\sigma$; (b) $A(+), L(+)$, and $\sigma(+) ;(\mathrm{c}) A(>x \mathrm{~km} / \mathrm{h})$
Table 3. Consistency Parameters

\begin{tabular}{ll}
\hline Parameter & \multicolumn{1}{c}{ Equation } \\
\hline 1 & $\sqrt{\frac{A(+) \cdot \sigma}{L}(\mathrm{~km} / \mathrm{h})}$ \\
3 & $\sqrt{\frac{A \cdot \sigma}{L}}(\mathrm{~km} / \mathrm{h})$ \\
4 & $\frac{A(+)}{L(+)}(\mathrm{km} / \mathrm{h})$ \\
5 & $\frac{A(>10 \mathrm{~km} / \mathrm{h})}{L}(\mathrm{~km} / \mathrm{h})$ \\
6 & $\frac{A(>15 \mathrm{~km} / \mathrm{h})}{L}(\mathrm{~km} / \mathrm{h})$ \\
7 & $\frac{A(>20 \mathrm{~km} / \mathrm{h})}{L}(\mathrm{~km} / \mathrm{h})$ \\
8 & $\sqrt{\frac{A(+) \cdot \sigma(+)}{L(+)}}(\mathrm{km} / \mathrm{h})$ \\
& $\sqrt{\frac{A(+) \cdot \sigma}{L(+)}(\mathrm{km} / \mathrm{h})}$
\end{tabular}

- $L(+)(\mathrm{m})$ : length of the road segment considering only the positives differences;

- $\sigma(+)(\mathrm{km} / \mathrm{h})$ : standard deviation of the difference between $V_{i}$ and $V_{85}$ considering only the positive differences; and

- $A(>x \mathrm{~km} / \mathrm{h})(\mathrm{m} \cdot \mathrm{km} / \mathrm{h}):$ area bounded when the difference between $V_{i}$ and $V_{85}$ is higher than $x \mathrm{~km} / \mathrm{h}$.

Table 3 summarizes the proposed consistency parameters. All parameters are expressed in speed terms. This is an important advantage in relation to other consistency models because the model interpretation is easier. Regarding the variables, the higher the value of the area or standard deviation, the lower the consistency because it means that the difference between both speeds is higher. On the other hand, when two road segments present the same area, the higher the length, the higher the consistency. Therefore, the level of consistency is greater as the value of any parameter is lower.

These parameters were calculated for each homogeneous road segment as the average between the values obtained for the forward and backward directions.

\section{Results}

\section{Functional Form and Goodness-of-Fit Measures}

The consistency parameter will be determined by examining its relationship to road crashes. Following common practice (Lord and Mannering 2010), generalized linear modeling techniques were used to fit a safety performance function that relates exposure and consistency to the number of crashes. A negative binomial distribution was assumed because it is a good solution with over dispersed count data (Lord and Mannering 2010). Only crashes with victims were considered, as stated previously. In addition, crashes of 10 years were considered to reduce the variability of these data. Thus, the safety performance function expression is as follows:

$$
Y_{i, 10}=e^{\beta_{0}} \cdot L^{\beta_{1}} \cdot A A D T^{\beta_{2}} \cdot e^{\beta_{3} \cdot C}
$$

where $Y_{i, 10}=$ accidents with victims of the road segment in 10 years; $\beta_{i}=$ regression coefficients; $L=$ length of the road 
segment $(\mathrm{km}) ; A A D T=$ average annual daily traffic volume $(\mathrm{vpd})$; and $C=$ consistency parameter $(\mathrm{km} / \mathrm{h})$.

Exposure parameters ( $A A D T$ and $L)$ are introduced as elasticity terms, which enables an easy interpretation of their behavior. Hence, it will be possible to determine how crash rates are affected depending on traffic volume and road segment length. In case the road length has no influence on crash rates, the $\beta_{1}$ coefficient will be automatically set to 1 .

The Akaike information criterion (AIC) was given in all regressions as a measure of the goodness of fit. This index is based on information theory and allows selecting a model from a set of models. In this regard, this criterion seeks a model that has a good fit with few parameters. A smaller AIC value indicates a better model. It is defined as

$$
A I C=-2 \cdot \log (\text { likelihood })+2 K
$$

where $\log ($ likelihood $)=\log$-likelihood at its maximum point of the model estimated; and $K=$ number of parameters in the model.

The quality of fit was also assessed from the cumulative residuals (CURE) plots (Hauer and Bamfo 1997; Lord and Persaud 2000). This method consists of plotting the cumulative residuals for each independent variable. The aim is to graphically observe how well the function fits the data set. The CURE method has the advantage of not being dependent on the number of observations. In general, a good cumulative residuals plot is one that oscillates around 0 . Thus, a good fit is given when the residuals do not stray beyond the $\pm 2 \sigma^{*}$ boundaries.

\section{Exposure Influence}

It is well known that crashes are highly affected by the exposure. Indeed, several previous researchers have developed safety performance functions that only depend on the exposure (Cafiso et al. 2010; Harwood et al. 2000). Thus, a first calibration is performed only with the exposure. This will allow determination of how the following consistency parameters affect the predictions, and whether it is worthwhile to consider them.

The expression of accidents in terms of exposure is

$$
Y_{i, 10}=e^{\beta_{0}} \cdot L^{\beta_{1}} \cdot A A D T^{\beta_{2}}
$$

where $Y_{i, 10}=$ accidents with victims of the road segment in 10 years; $\beta_{i}=$ regression coefficients; $L=$ length of the road segment $(\mathrm{km})$; and $A A D T=$ average annual daily traffic (vpd).

Table 4 shows the outcomes of the adjustment. As expected, all parameters are statistically significant. The $A A D T$ estimate is close to 1 . This indicates that the number of crashes is linearly affected by the traffic volume under consideration. On the other hand, the length estimate is lower than 1 . This indicates that a higher length results in lower crash rates.

The AIC of this model will be compared to those determined for the consistency models.

Table 4. Statistical Adjustment-Model Considering Exposure

\begin{tabular}{lcrc}
\hline Variable & $\begin{array}{c}\text { Regression } \\
\text { coefficient }\end{array}$ & Estimate & $\operatorname{Pr}(>|z|)$ \\
\hline Constant & $\beta_{0}$ & -7.2212 & $3.08 \times 10^{-14}$ \\
Natural logarithm of $L$ & $\beta_{1}$ & 0.7070 & $1.27 \times 10^{-6}$ \\
Natural logarithm of $A A D T$ & $\beta_{2}$ & 1.0307 & $<2 \times 10^{-16}$ \\
Overdispersion parameter $=0.34282$ & & \\
$A I C=557.92$ & & \\
\hline
\end{tabular}

\section{Consistency Influence}

A total of 480 safety performance functions were calibrated by combining 60 inertial speed profile variations and eight consistency parameters.

All models were arranged considering their $A I C$ values. In this regard, Table 5 shows the 25 models with the lowest $A I C$ values. It could be observed that Parameter 7 was the most important. This parameter was calculated from several variables that considered only the positive difference between the inertial operating speed and operating speed (Table 3). As mentioned previously, a positive difference means that drivers' expectations are violated, which produces an increase of the likelihood of crash occurrence.

The best models presented consistency parameters that were calculated considering linear or parabolic weighting distributions. In addition, the $A I C$ values were lower than the $A I C$ value considering only the exposure $(A I C=557.92)$. Therefore, all hypotheses of the study were verified. In this regard, the last meters driven have higher importance on the driver's behavior.

However, the evolution of the $A I C$ value was also analyzed according to each parameter and weighting distribution. Its objective was to identify the best model more properly. As an example, Figs. 6 and 7 show the trend of the $A I C$ value considering Parameter 7 and the convex parabolic distribution, respectively.

In general, the lowest $A I C$ values were found for distances from 500 to $700 \mathrm{~m}$. Parameter 7 presented the best behavior as a consistency indicator, so the proposed model was the 600PV7 one (Table 6). This model uses Consistency Parameter 7 (Table 3), which was obtained from the inertial operating speed profile considering $600 \mathrm{~m}$ and a concave parabolic distribution.

Finally, the model was validated by means of CURE plots (Fig. 8). It can be observed that the plots against each explanatory variable do not stray beyond the $\pm 2 \sigma^{*}$ boundaries, apart from a few points where the $A A D T$ or $C$ is high. It is mainly because of the few

Table 5. Ranking of the Models according to the AIC Value

\begin{tabular}{llccc}
\hline Model & Parameter & $\begin{array}{c}\text { Length } \\
(\mathrm{m})\end{array}$ & $\begin{array}{c}\text { Weighting } \\
\text { distribution }\end{array}$ & AIC \\
\hline 600PV7 & Parameter 7 & 600 & Concave & 548.15 \\
600L7 & Parameter 7 & 600 & Linear & 548.18 \\
600L3 & Parameter 3 & 600 & Linear & 548.34 \\
600PV3 & Parameter 3 & 600 & Concave & 548.37 \\
500PX3 & Parameter 3 & 500 & Convex & 548.38 \\
500PX8 & Parameter 8 & 500 & Convex & 548.38 \\
200C4 & Parameter 4 & 200 & Constant & 548.39 \\
700PV7 & Parameter 7 & 700 & Concave & 548.42 \\
500PX7 & Parameter 7 & 500 & Convex & 548.52 \\
600PV8 & Parameter 8 & 600 & Concave & 548.54 \\
600L8 & Parameter 8 & 600 & Linear & 548.69 \\
500L7 & Parameter 7 & 500 & Linear & 548.79 \\
500L8 & Parameter 8 & 500 & Linear & 548.82 \\
700PV8 & Parameter 8 & 700 & Concave & 548.84 \\
800PV7 & Parameter 7 & 800 & Concave & 548.86 \\
200 PX4 & Parameter 4 & 200 & Convex & 548.98 \\
700PV3 & Parameter 3 & 700 & Concave & 549.04 \\
300L4 & Parameter 4 & 300 & Linear & 549.05 \\
500PV7 & Parameter 7 & 500 & Concave & 549.09 \\
300 PX7 & Parameter 7 & 300 & Convex & 549.09 \\
700PV1 & Parameter 1 & 700 & Concave & 549.17 \\
600PV1 & Parameter 1 & 600 & Concave & 549.21 \\
500PV8 & Parameter 8 & 500 & Concave & 549.21 \\
300PX8 & Parameter 8 & 300 & Convex & 549.29 \\
700L7 & Parameter 7 & 700 & Linear & 549.3 \\
\hline
\end{tabular}




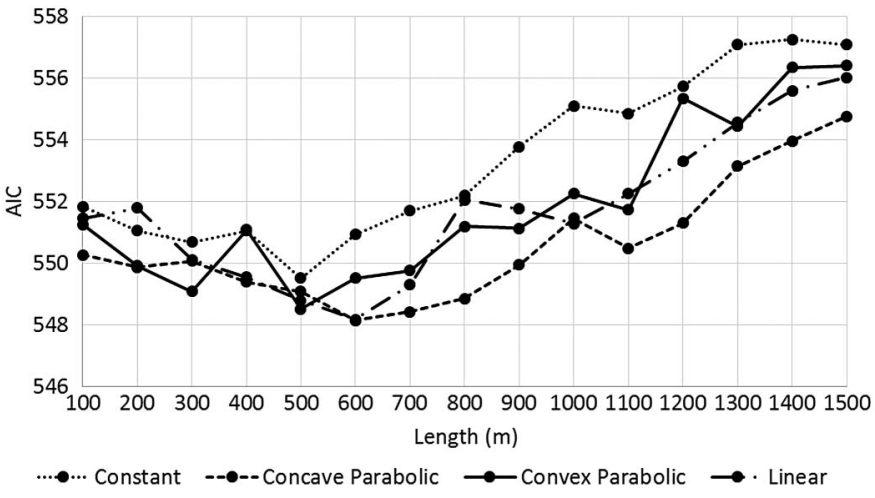

Fig. 6. Evolution of the $A I C$ value considering the Parameter 7

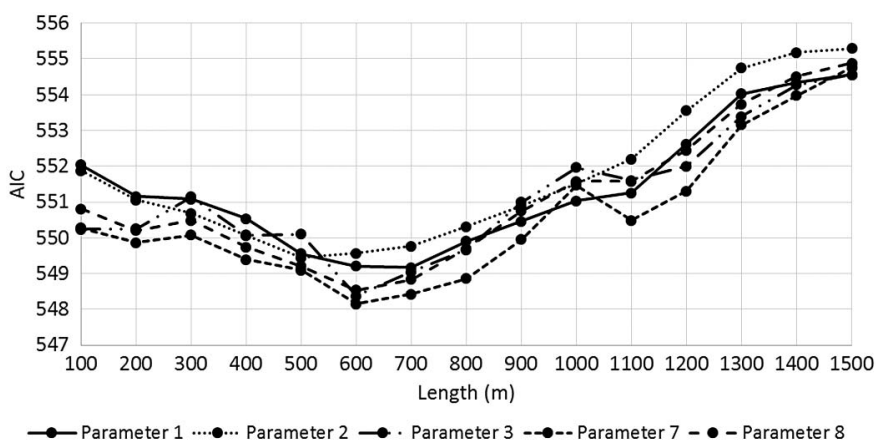

Fig. 7. Evolution of the $A I C$ value considering the concave parabolic distribution

Table 6. Statistical Adjustment-Model Considering Consistency

\begin{tabular}{lcrr}
\hline Variable & $\begin{array}{c}\text { Regression } \\
\text { coefficient }\end{array}$ & Estimate & $\operatorname{Pr}(>|z|)$ \\
\hline Constant & $\beta_{0}$ & -8.63431 & $<2 \times 10^{-16}$ \\
Natural logarithm of $L$ & $\beta_{1}$ & 1.09153 & $3.21 \times 10^{-10}$ \\
Natural logarithm of $A A D T$ & $\beta_{2}$ & 1.03547 & $<2 \times 10^{-16}$ \\
Consistency $(C)$ & $\beta_{3}$ & 0.18128 & 0.000376 \\
Overdispersion parameter $=0.2932$ & & \\
AIC $=548.15$ & & \\
\hline
\end{tabular}

available data for high traffic volumes and road segments with very poor consistency. In these situations, the proposed model tends to underestimate the number of accidents. Therefore, it is recommended to use the new consistency model for road segments that present a traffic volume lower than 13,500 vpd. Despite this, the new consistency model is a useful tool for engineers to estimate the number of crashes in Italian two-lane rural roads.

\section{Discussion}

\section{Inertial Operating Speed}

Drivers' expectations have been modeled from inertial operating speed, which was defined as the weighted average operating speed. Different distances and weighting distributions were studied to identify how the inertial operating speed should be estimated. As a result, this speed should be calculated at each point of the alignment considering a concave parabolic distribution and $600 \mathrm{~m}$, which supports the hypotheses of the study.

The constant distribution, which had been used by other authors previously (García et al. 2013b; Montella and Imbriani 2015), presented worse results than linear and parabolic distributions. This phenomenon highlights that drivers' behavior is more influenced by the last meters driven, which is consistent with short-term memory.

\section{Effect of the Consistency Parameter on Road Crashes}

The consistency parameter is valid for estimating the number of crashes. This parameter is defined as the square root of the area bounded by the difference between $V_{i}$ and $V_{85}[A(+)]$ divided by the length of the road segment $[L(+)]$ and multiplied by the standard deviation of the difference between both speeds $[\sigma(+)]$, considering only the positives differences. The higher the consistency parameter, the higher the crash rate (Fig. 9).

A positive difference between $V_{i}$ and $V_{85}$ means that drivers' expectations are violated. Therefore, a higher crash rate is expected. Thus, for a given $A(+)$, a higher length and a lower $\sigma(+)$ lead to a lower crash rate, i.e., a good consistency. Likewise, for a given $L(+)$, a higher $A(+)$ or $\sigma(+)$ leads to a higher crash rate, i.e., a poor consistency. All of this can be observed in Fig. 10, where the crash rate is shown as the size of the circles.

\section{Comparison with Previous Global Consistency Models}

The new consistency model has been compared with the global consistency models developed by Polus and Mattar-Habib (2004), Garach et al. (2014), and Camacho-Torregrosa (2015).

First, different safety performance functions were calibrated considering the consistency parameters proposed by the authors. Table 7 shows the outcomes of the adjustment. It can be observed that the $A I C$ values were higher than the $A I C$ values of the new model (Table 6). Therefore, the new consistency parameter presents a closer relationship with the crashes than the previous models.

Finally, a graphic was developed to analyze the relationship between the number of observed and predicted crashes (Fig. 11). As a result, the behavior of all models was similar. The coefficient of determination of the linear model considering the new consistency parameter $(60 \%)$ was higher than the models developed from the consistency parameters of Polus and Mattar-Habib $(59 \%)$ and Garach et al. (57\%), but lower than the model developed by Camacho-Torregrosa (62\%). In addition, all models presented similar values for the root-mean-square error (RMSE) and the mean absolute error (MAE) and tended to underestimate the number of crashes when the number of observed crashes was high.

Therefore, the new consistency parameter can better represent the phenomenon, enabling a more accurate estimation of crashes than the previous global consistency models.

\section{Consistency Model Application}

The new global consistency model can be used to evaluate road safety in the design or improvement stage. For this, the first step is the estimation of the operating speed profile. In this regard, Marchionna and Perco's model is recommended because it was developed using Italian two-lane rural road data. Next, the inertial operating speed is calculated at each point of the alignment as the weighted average operating speed according to a concave parabolic distribution considering a 600-m-long section. Finally, the consistency parameter is determined from the difference between the inertial operating speed profile and the operating speed profile, 

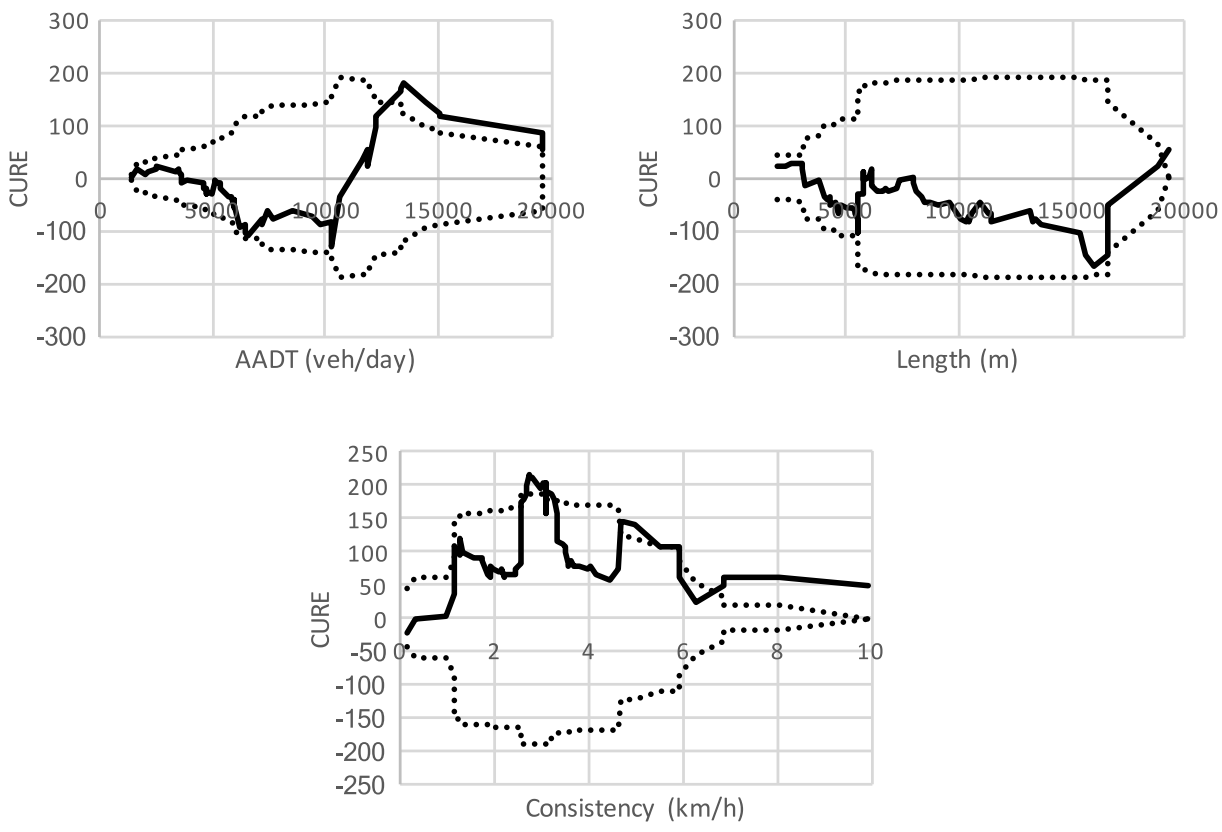

Fig. 8. CURE plots

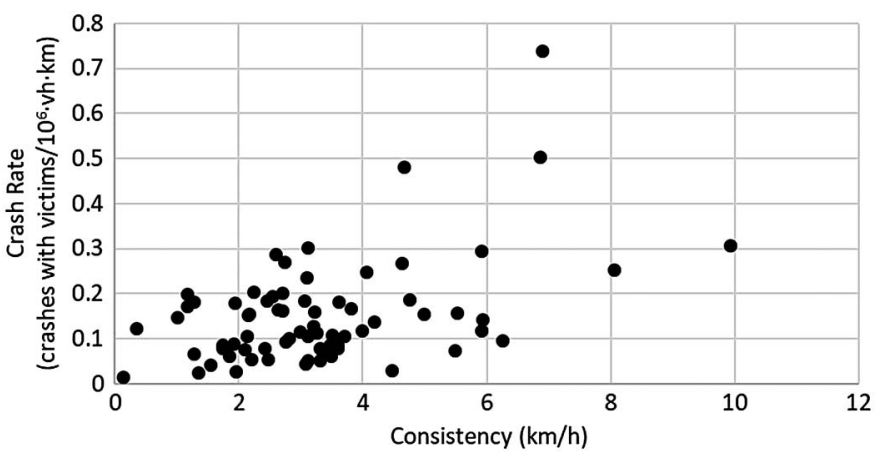

Fig. 9. Relationship between the crash rate and the consistency parameter

allowing engineers to estimate the number of crashes by means of the following safety performance function:

$$
Y_{i, 10}=e^{-8.63431} \cdot L^{1.09153} \cdot A A D T^{1.03547} \cdot e^{0.18128 \cdot C}
$$

where $Y_{i, 10}=$ accidents with victims of the road segment in 10 years; $L=$ length of the road segment $(\mathrm{km}) ; A A D T=$ average annual daily traffic (vpd); and $C=$ consistency parameter $(\mathrm{km} / \mathrm{h})$ defined as:

$$
C=\sqrt{\frac{A(+) \cdot \sigma(+)}{L(+)}}
$$
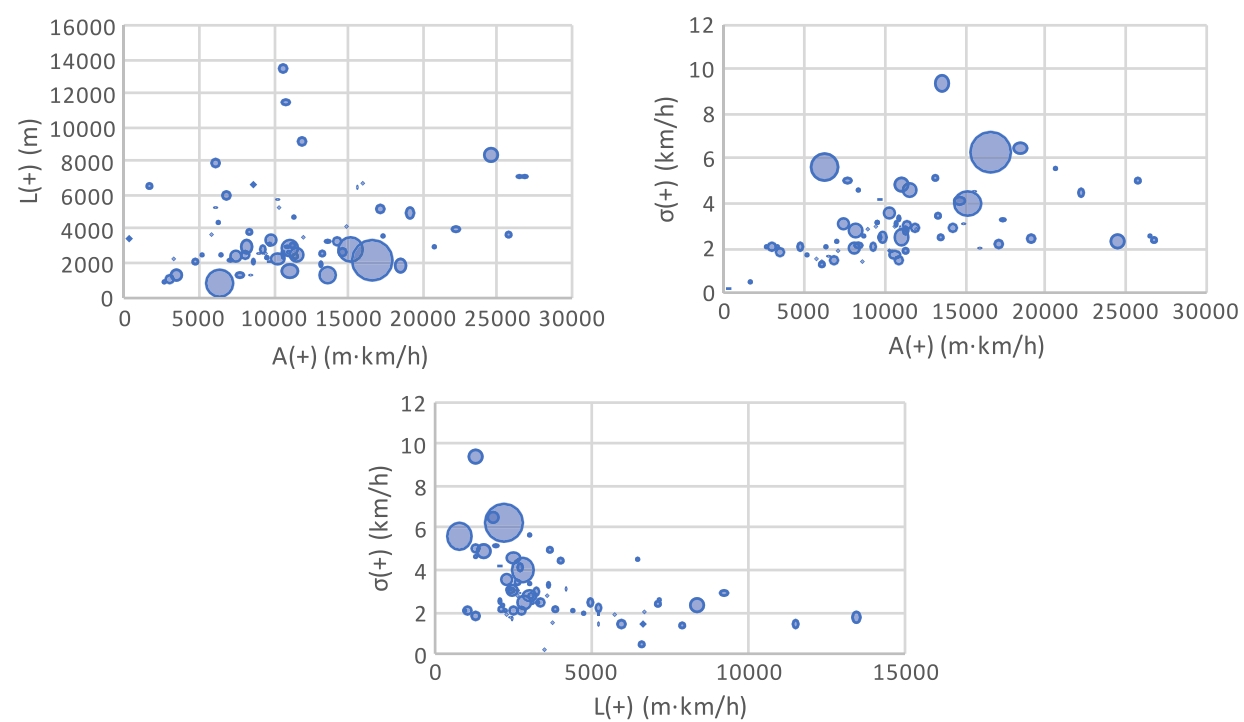

Fig. 10. Relationship between consistency variables and crash rate 
Table 7. Statistical Adjustment—Previous Global Consistency Models

\begin{tabular}{lcrc}
\hline Variable & $\begin{array}{c}\text { Regression } \\
\text { coefficient }\end{array}$ & Estimate & $\operatorname{Pr}(>|z|)$ \\
\hline Polus and Mattar-Habib (2004) & & & \\
Constant & $\beta_{0}$ & -6.9582 & $<2 \times 10^{-16}$ \\
Natural logarithm of $L$ & $\beta_{1}$ & 0.9458 & $4.26 \times 10^{-8}$ \\
Natural logarithm of $A A D T$ & $\beta_{2}$ & 0.9929 & $<2 \times 10^{-16}$ \\
Consistency $(C)$ & $\beta_{3}$ & -0.3352 & 0.00943 \\
Overdispersion parameter $=0.31736$ & & \\
AIC =553.48 & & & \\
Garach et al. (2014) & $\beta_{0}$ & -7.0805 & $<2 \times 10^{-16}$ \\
Constant & $\beta_{1}$ & 0.9050 & $9.58 \times 10^{-8}$ \\
Natural logarithm of $L$ & $\beta_{2}$ & 1.0146 & $<2 \times 10^{-16}$ \\
Natural logarithm of $A A D T$ & $\beta_{3}$ & -0.2917 & 0.0169 \\
Consistency $(C)$ & & \\
Overdispersion parameter $=0.32468$ & & \\
AIC = 554.87 & & & \\
Camacho-Torregrosa $(2015)$ & $\beta_{0}$ & -6.7761 & $5.21 \times 10^{-16}$ \\
Constant & $\beta_{1}$ & 0.9206 & $1.94 \times 10^{-7}$ \\
Natural logarithm of $L$ & $\beta_{2}$ & 1.0335 & $<2 \times 10^{-16}$ \\
Natural logarithm of $A A D T$ & $\beta_{3}$ & -0.2221 & 0.0379 \\
Consistency $(C)$ & & \\
Overdispersion parameter $=0.32541$ & & \\
AIC = 555.75 & & & \\
\hline
\end{tabular}

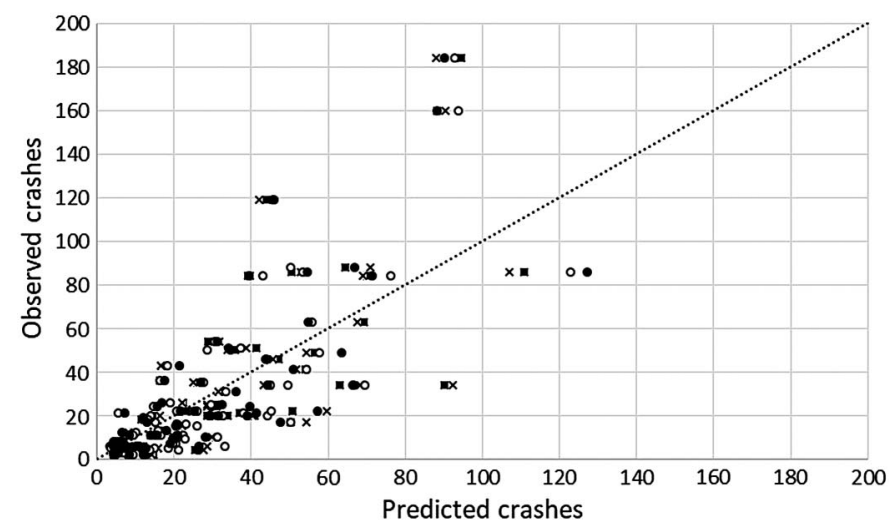

m Polus and Mattar-Habib (2004) × Garach et al. (2014)

- Camacho-Torregrosa (2015) • New model

Fig. 11. Observed and predicted accidents considering different consistency parameters

where $A(+), L(+)$, and $\sigma(+)$ are consistency variables defined previously.

This safety performance function based on the new consistency model is a useful tool for engineers to compare and sort different design proposals, maximizing road safety. Nevertheless, it is recommended to use this model complementarily with a local consistency model. The global model will allow estimating the number of crashes on an entire road segment, whereas the local model will indicate where the most hazardous points are located.

\section{Conclusions}

A new global consistency model has been presented in this paper. A set of 71 homogeneous two-lane rural road segments were used in the analysis. As a result, approximately $550 \mathrm{~km}$ were recreated.
The new model was developed from the difference between the inertial operating speed profile and the operating speed profile. In this regard, $V_{i}$ represents drivers' expectations, whereas $V_{85}$ represents road behavior. The higher the difference between both speeds, the higher the crash rate because this means that drivers' expectations are violated.

Different distances and weighting distributions were studied to identify how inertial operating speed should be calculated. In addition, eight consistency parameters were proposed for the analysis. As a result, 480 safety performance functions were calibrated.

Most safety performance functions that incorporate a consistency parameter showed lower AIC values than the safety performance function considering only the exposure. Therefore, the level of consistency significantly influences the occurrence of crashes.

The best model was the 600PV7 model, which used Consistency Parameter 7 (Table 3). This parameter was obtained from the inertial operating speed profile considering $600 \mathrm{~m}$ and a concave parabolic distribution, which was consistent with the shortterm memory behavior.

The new consistency parameter is defined as the square root of the area bounded by the difference between $V_{i}$ and $V_{85}[A(+)]$ divided by the length of the road segment $[L(+)]$ and multiplied by the standard deviation of the difference between both speeds $[\sigma(+)]$ considering only the positive differences. Thus, the lower the $A(+)$ or $\sigma(+)$ and the higher the $L(+)$, the higher the consistency.

The new safety performance function was compared with several safety performance functions calibrated from the consistency parameters proposed by Polus and Mattar-Habib (2004), Garach et al. (2014), and Camacho-Torregrosa (2015). As a result, the new model showed the lowest $A I C$ value and a closer relationship with the observed crashes.

Therefore, the new consistency parameter based on the geometric design consistency concept describes the phenomenon better than the previous global consistency models. Thus, the new safety performance function is a useful tool for the engineers that allows estimating the number of crashes and incorporates road safety into the geometric design of both new Italian two-lane rural roads and improvements of existing highways.

\section{Acknowledgments}

This research was subsidized by the Spanish Ministry of Economy and Competitiveness through "Ayudas a la movilidad predoctoral para la realización de estancias breves en centros de I+D 2015.” The study presented in this paper is also part of the research project titled "CASEFU_Estudio experimental de la funcionalidad y seguridad de las carreteras convencionales" (TRA2013-42578-P), subsidized by the Spanish Ministry of Economy and Competitiveness and the European Social Fund. In addition, the authors would like to thank the Azienda Nazionale Autonoma delle Strade (ANAS) and the Automobile Club Italia (ACI), which provided traffic and crash data, respectively.

\section{References}

Anderson, I., Bauer, K., Harwood, D., and Fitzpatrick, K. (1999). "Relationship to safety of geometric design consistency measures for rural two-lane highways." Transp. Res. Rec., 1658, 43-51.

Awatta, M., Hassan, Y., and Sayed, T. (2006). "Quantitative evaluation of highway safety performance based on design consistency." Advances in transportation studies, Vol. 9, Roma Tre Univ., Rome.

Cafiso, S., Di Graziano, A., Di Silvestro, G., La Cava, G., and Persaud, B. (2010). "Development of comprehensive accident models for two-lane 
rural highways using exposure, geometry, consistency and context variables." Accid. Anal. Prev., 42(4), 1072-1079.

Camacho-Torregrosa, F. J. (2015). "Development and calibration of a global geometric design consistency model for two-lane rural highways, based on the use of continuous operating speed profiles." Universitat Politècnica de València, Valencia, Spain.

Camacho-Torregrosa, F. J., Pérez-Zuriaga, A. M., Campoy-Ungría, J. M., García, A., and Tarko, A. P. (2015). "Use of heading direction for recreating the horizontal alignment of an existing road." Comput.-Aided Civ. Infrastruct. Eng., 30(4), 282-299.

European Transport Safety Council. (2015). Ranking EU progress on improving motorway safety, Vol. 28, Etterbeek, Belgium.

Garach, L., Calvo, F., Pasadas, M., and de Oña, J. (2014). "Proposal of a new global model of consistency: Application in two-lane rural highways in Spain.” J. Transp. Eng., 10.1061/(ASCE)TE.1943-5436 $.0000683,04014030$

Garcia, A., Llopis-Castello, D., Perez-Zuriaga, A. M., and CamachoTorregrosa, F. J. (2013a). "Homogeneous road segment identification based on inertial operating speed." Proc., 92nd Annual Meeting on Transportation Research Board, Washington, DC.

García, A., Llopis-Castelló, D., Camacho-Torregrosa, F. J., and PérezZuriaga, A. M. (2013b). "New consistency index based on inertial operating speed." Transp. Res. Rec., 2391, 105-112.

Gibreel, G. M., Easa, S. M., Hassan, Y., and El-Dimeery, I. A. (1999). "State of the art of highway geometric design consistency." J. Transp. Eng., 10.1061/(ASCE)0733-947X(1999)125:4(305), 305-313.

Harwood, D. W., Council, F. M., Hauer, E., Hughes, W. E., and Vogt, A. (2000). "Prediction of the expected safety performance of rural two-lane highways." No. FHWA-RD-99-207, Federal Highway Administration, Washington, DC.

Hauer, E., and Bamfo, J. (1997). "Two tools for finding what function links the dependent variable to the explanatory variables." Proc., 10th ICTCT Workshop, Lund, Sweden.

Kanellaidis, G., Golias, J., and Efstathiadis, S. (1990). "Drivers' speed behaviour on rural road curves." Traffic Eng. Control, 31(7-8), 414-415.

Lamm, R., Psarianos, B., and Mailaender, T. (1999). Highway design and traffic safety engineering handbook, McGraw-Hill, New York.

Leisch, J. E., and Leisch, J. P. (1977). "New concepts in design-speed application." Transp. Res. Rec., 631, 4-14.

Lord, D., and Mannering, F. (2010). "The statistical analysis of crashfrequency data: A review and assessment of methodological alternatives.” Transp. Res. Part A, 44(5), 291-305.
Lord, D., and Persaud, B. (2000). "Accident prediction models with and without trend: Application of the generalized estimating equations procedure." Transp. Res. Rec., 1717, 102-108.

Marchionna, A., and Perco, P. (2008). "Operating speed-profile prediction model for two-lane rural roads in the Italian context." Adv. Transp. Stud., 2008(14), 57-68.

Mattar-Habib, C., Polus, A., and Farah, H. (2008). "Further evaluation of the relationship between enhanced consistency model and safety of two-lane rural roads in Israel and Germany." Eur. J. Transp. Infrastruct. Res., 4(8), 320-332.

Montella, A., Colantuoni, L., and Lamberti, R. (2008). "Crash prediction models for rural motorways." Transp. Res. Rec., 2083, 180-189.

Montella, A., and Imbriani, L. L. (2015). "Safety performance functions incorporating design consistency variables." Accid. Anal. Prev., 74, 133-144.

Ng, J. C., and Sayed, T. (2004). "Effect of geometric design consistency on road safety." Can. J. Civ. Eng., 31(2), 218-227.

Polus, A., and Mattar-Habib, C. (2004). "New consistency model for rural highways and its relationship to safety." J. Transp. Eng., 10.1061 /(ASCE)0733-947X(2004)130:3(286), 286-293.

Praticò, F. G., and Giunta, M. (2012). "Quantifying the effect of present, past and oncoming alignment on the operating speeds of a two-lane rural road." Baltic J. Road Bridge Eng., 7(3), 179-188.

Quddus, M. (2013). "Exploring the relationship between average speed, speed variation, and accident rates using spatial statistical models and GIS." J. Transp. Safety Security, 5(1), 27-45.

Resende, P., and Benekohal, R. (1997). "Effects of roadway section length on accident modeling." Traffic congestion and traffic safety in the 21st century: Challenges, innovations, and opportunities, ASCE, New York, 403-409.

Revlin, R. (2012). Cognition: Theory and practice, Palgrave Macmillan, Basingstoke, U.K.

Treat, J. R., Tumbas, N. S., McDonald, S. T., Shinar, D., and Hume, R. D. (1979). "Tri-level study of the causes of traffic accidents. Executive summary." Rep. No. DOT-HS-034-3-535-79-TAC(S), Institute for Research in Public Safety, Bloomington, IN.

WHO (World Health Organization). (2015). Global status report on road safety 2015, Vol. 19, Geneva, 340.

Wu, K. F., Donnell, E. T., Himes, S. C., and Sasidharan, L. (2013). "Exploring the association between traffic safety and geometric design consistency based on vehicle speed metrics." J. Transp. Eng., 10.1061 /(ASCE)TE.1943-5436.0000553, 738-748. 